\title{
MIPSTR: a method for multiplex genotyping of germline and somatic STR variation across many individuals
}

\author{
Keisha D. Carlson, ${ }^{1}$ Peter H. Sudmant, ${ }^{1}$ Maximilian O. Press, ${ }^{1}$ Evan E. Eichler, ${ }^{1,2}$ \\ Jay Shendure, ${ }^{1}$ and Christine Queitsch ${ }^{1}$ \\ ${ }^{1}$ Department of Genome Sciences, University of Washington, Seattle, Washington 98195, USA; ${ }^{2}$ Howard Hughes Medical Institute, \\ University of Washington School of Medicine, Seattle, Washington 98195, USA
}

\begin{abstract}
Short tandem repeats (STRs) are highly mutable genetic elements that often reside in regulatory and coding DNA. The cumulative evidence of genetic studies on individual STRs suggests that STR variation profoundly affects phenotype and contributes to trait heritability. Despite recent advances in sequencing technology, STR variation has remained largely inaccessible across many individuals compared to single nucleotide variation or copy number variation. STR genotyping with short-read sequence data is confounded by (1) the difficulty of uniquely mapping short, low-complexity reads; and (2) the high rate of STR amplification stutter. Here, we present MIPSTR, a robust, scalable, and affordable method that addresses these challenges. MIPSTR uses targeted capture of STR loci by single-molecule Molecular Inversion Probes (smMIPs) and a unique mapping strategy. Targeted capture and our mapping strategy resolve the first challenge; the use of single molecule information resolves the second challenge. Unlike previous methods, MIPSTR is capable of distinguishing technical error due to amplification stutter from somatic STR mutations. In proof-of-principle experiments, we use MIPSTR to determine germline STR genotypes for 102 STR loci with high accuracy across diverse populations of the plant $A$. thaliana. We show that putatively functional STRs may be identified by deviation from predicted STR variation and by association with quantitative phenotypes. Using DNA mixing experiments and a mutant deficient in DNA repair, we demonstrate that MIPSTR can detect low-frequency somatic STR variants. MIPSTR is applicable to any organism with a high-quality reference genome and is scalable to genotyping many thousands of STR loci in thousands of individuals.
\end{abstract}

[Supplemental material is available for this article.]

Variation in short tandem repeats (STRs), which are also known as microsatellites, significantly contributes to phenotypic variation, evolutionary adaptation, and human disease (Gemayel et al. 2012). STRs consist of short (2-10 bp) DNA sequences (units) that are repeated head to tail. The presence of multiple identical or nearly identical adjacent sequence units causes frequent errors in recombination and replication, resulting in loss or gain of units. Consequently, STR mutation rates are 10-10,000 times higher than mutation rates of nonrepetitive loci (Legendre et al. 2007; Eckert and Hile 2009).

In spite of their hypervariability, STRs frequently reside in functional DNA, including coding and regulatory regions. STRs are estimated to be present in $6 \%$ of human coding regions (O'Dushlaine et al. 2005; Mularoni et al. 2006), highlighting the potential of STR variation to affect disease risk and other complex traits. Coding STRs that vary among humans tend to reside in genes affecting transcription and neural development (Molla et al. 2009). Several severe genetic diseases, including the trinucleotide expansion disorders Huntington's and Spinocerebellar Ataxias (SCA), are a consequence of extended STR alleles that act as dominant mutations (Gatchel and Zoghbi 2005). The severity of STR expansion disorders would suggest that natural selection should remove STRs from functional genomic regions, but some,

Corresponding author: queitsch@u.washington.edu

Article published online before print. Article, supplemental material, and publication date are at http://www.genome.org/cgi/doi/10.1101/gr.182212.114. for example the pre-expansion STR allele in ATXN2, are maintained by selection (Yu et al. 2005).

Model organism studies have demonstrated significant functional consequences of even subtle unit number variation in select STRs in plants, fungi, flies, voles, dogs, and fish, among other organisms (Sawyer et al. 1997; Fondon and Garner 2004; Hammock and Young 2005; Michael et al. 2007; Undurraga et al. 2012; Scarpino et al. 2013; Rosas et al. 2014). Similar to humans, STR-containing genes in these organisms tend to be regulatory genes functioning in transcription, development, and sensing environmental factors (Fondon and Garner 2004; Verstrepen et al. 2005). Adding or subtracting a single STR unit can have dramatic phenotypic effects, such as in the polyglutamine-encoding STR in the circadian clock gene ELF3 in Arabidopsis thaliana (Undurraga et al. 2012). STR unit number can show striking nonlinear relationships with phenotype, which may in part be due to extensive epistatic interactions with other loci (Peixoto et al. 1998; Butler et al. 2007; Undurraga et al. 2012). Based on existing evidence, STR variation likely comprises an important component of the genotype-phenotype map (e.g., STRs are a viable explanation for some component of the "missing heritability" of genome-wide association studies) (Press et al. 2014); yet due to

(C) 2015 Carlson et al. This article is distributed exclusively by Cold Spring Harbor Laboratory Press for the first six months after the full-issue publication date (see http://genome.cshlp.org/site/misc/terms.xhtml). After six months, it is available under a Creative Commons License (Attribution-NonCommercial 4.0 International), as described at http://creativecommons.org/licenses/by$\mathrm{nc} / 4.0 /$. 
technological difficulties in genotyping STRs, this component has remained largely undefined.

STRs have almost entirely escaped genome-wide assessment across many individuals due to the complexities of uniquely mapping short, repetitive sequencing reads and the inherently high error rate of STR amplification (i.e., amplification stutter). Although traditional Sanger sequencing accurately assesses STR genotypes, this costly method is severely limited in throughput. Hence, it is not well suited for genotyping STRs across many individuals. Nevertheless, when researchers compared the three Sangersequenced human genomes (including the reference genome), they found significant STR variation (Molla et al. 2009). In the thousands of short-read sequenced genomes available for a variety of organisms, we would therefore expect to see an even larger degree of STR variation among these many individuals. However, STR variation is typically excluded or misreported for genomes sequenced with short reads. Recently, several tools have been developed to estimate STR unit number from short read sequencing data (Gymrek et al. 2012; Highnam et al. 2013; Tae et al. 2013). These tools rely on the use of STR-spanning reads with unique flanking regions to improve mappability and ascertain STR unit number. This restriction imposes size limits (read lengths in extant data are generally $101 \mathrm{bp}$ or less) and greatly reduces coverage of informative reads (Supplemental Fig. 1). For example, when assessing the genotype of an STR locus of $\sim 30 \mathrm{bp}$ for a genome sequenced with 101-bp reads at $5 \times$ coverage, one will have to rely on fewer than three STR-spanning reads on average. Moreover, these tools model technical error due to amplification stutter based on STR genotypes from sequenced homozygous or haploid genomes, ignoring the expected diversity of somatic alleles within individuals. These probabilistic models lose applicability in practice because STR genotype calls are made with as few as one or two STR-spanning reads. Another recent method uses paired-end sequencing reads to infer variation at STR loci, similar to previous methods to detect large insertions and deletions (Chen et al. 2009; Hajirasouliha et al. 2010; Qi and Zhao 2011; Grimm et al. 2013). Due to the resolution limits of gel size selection, this method infers only whether STRs are variable rather than calling STR unit number genotypes (Cao et al. 2014). Thus, the comprehensive assessment of accurate STR genotypes from short-read sequencing data has remained a largely intractable problem.

Vast numbers of genomes, including genomes of hundreds of A. thaliana strains, have been generated with 36- to 64-bp read lengths (Cao et al. 2011; Gan et al. 2011) that are too short for the aforementioned tools. The existing read lengths and coverage depths of these genomes are sufficient to call most single nucleotide variants (SNVs), but insufficient to understand STR variation. It would be inefficient and costly to resequence whole genomes of hundreds of individuals or strains with sufficient depth and the longer reads necessary to understand STR variation $(\sim 150-300$ bp, $>30 \times$ coverage) when STRs only make up a small portion of the genome.

The challenges of STR genotyping can be addressed by targeted STR capture to increase the number of STR-spanning reads combined with a sequencing technology that accommodates longer reads to improve mappability and STR genotype calling. Such strategies were recently applied to the human genome, using STRtargeted microarray capture or RNA probe capture prior to sequencing (Guilmatre et al. 2013; Duitama et al. 2014). However, these STR capture methods produced only limited enrichment for STR-containing reads with flanking sequence, i.e., $2.2 \%$ of mappable reads (Guilmatre et al. 2013) and 25\% of mappable reads (Duitama et al. 2014), and only marginally improved STR coverage for unit number calls (Table 1).

Here, we address the major obstacles of STR genotyping with a robust, scalable, and inexpensive method, MIPSTR. MIPSTR combines STR capture via single-molecule Molecular Inversion Probes (smMIPs) (Hiatt et al. 2013) with midsize sequencing reads (250 bp) and a unique mapping strategy. In proof-of-principle experiments, we captured and sequenced STRs genome-wide in diverse $A$. thaliana populations, called germline STR genotypes with high accuracy, and quantified technical error with singlemolecule information. Moreover, enabled by single-molecule degenerate sequence tags, we demonstrate that MIPSTR can capture the same STR locus from thousands of different cells, thereby enabling detection of somatic STR variants with high sensitivity.

\section{Results}

\section{Single molecule capture strategy yields highly accurate STR germline genotypes}

We used single-molecule Molecular Inversion Probes (smMIPs) (Hiatt et al. 2013) to capture STRs, thereby maximizing the number of STR-spanning, informative reads. In a proof-of-principle experiment, we targeted 102 STRs across the genome of the model

Table 1. Technologies for assessing STR variation by targeted capture and high-throughput sequencing

\begin{tabular}{|c|c|c|c|c|c|c|c|}
\hline Name & $\begin{array}{l}\text { Sequencing and } \\
\text { analysis strategy }\end{array}$ & $\begin{array}{l}\text { Accepted } \\
\text { coverage }^{\mathrm{a}}\end{array}$ & $\begin{array}{l}\text { Reported } \\
\text { accuracy }\end{array}$ & $\begin{array}{l}\text { Reads mapped } \\
\text { to STR targets } \\
\text { (total targets) }\end{array}$ & $\begin{array}{l}\text { Efficiency of } \\
\text { mapped reads }\end{array}$ & $\begin{array}{l}\text { STR targets } \\
\text { successfully genotyped } \\
\text { (total number) }\end{array}$ & Reference \\
\hline Array capture & $\begin{array}{l}\text { Human, Illumina } \\
\text { HiSeq, RepeatSeq } \\
\text { (Highnam et al. } \\
\text { 2013) }\end{array}$ & Two reads & $88 \%-92 \%$ & $38.7 \%(7851)$ & $\begin{array}{l}6.5 \% \\
\text { informative } \\
\text { reads }\end{array}$ & $\begin{array}{l}\text { Average } 54.2 \% \text { of } \\
\text { targets across eight } \\
\text { samples }(33,947)\end{array}$ & $\begin{array}{l}\text { (Guilmatre } \\
\text { et al. } \\
\text { 2013) }\end{array}$ \\
\hline $\begin{array}{l}\text { SureSelect } \\
\text { RNA probe } \\
\text { capture }\end{array}$ & $\begin{array}{l}\text { Human, Roche } 454 \text {, } \\
\text { locally align flanking } \\
\text { regions }\end{array}$ & Four reads & $88 \%-95 \%$ & $\sim 60 \%(10,764)$ & $\begin{array}{l}40 \% \\
\text { informative } \\
\text { reads }\end{array}$ & $\begin{array}{l}30.1 \%-36.8 \% \text { of } \\
\text { targets in each of } \\
\text { seven samples } \\
(\sim 27,699)\end{array}$ & $\begin{array}{l}\text { (Duitama } \\
\text { et al. } \\
\text { 2014) }\end{array}$ \\
\hline $\begin{array}{l}\text { MIPSTR- } \\
\text { smMIP } \\
\text { capture }\end{array}$ & $\begin{array}{l}\text { A. thaliana, Illumina } \\
\text { MiSeq, map to } \\
\text { locus-specific } \\
\text { synthetic reference }\end{array}$ & Four reads & $94 \%-98 \%$ & $72 \%(100)$ & $\begin{array}{l}55 \%-64 \% \\
\text { informative } \\
\text { reads }\end{array}$ & $\begin{array}{l}\text { Average } 64 \% \text { of targets } \\
\text { across } 96 \text { samples } \\
\text { (6144) }\end{array}$ & \\
\hline
\end{tabular}

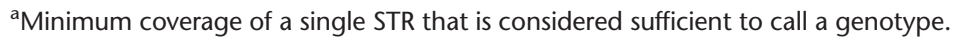


plant, $A$. thaliana, including exonic, intronic, regulatory (Sullivan et al. 2014), and intergenic tri- and hexa-nucleotide STRs (Supplemental Fig. 2; Supplemental Table 1). We chose tri- and hexa-nucleotide STRs because these occur commonly in protein-coding regions, allowing us to compare and contrast coding and noncoding STR variation. We first applied MIPSTR to the reference A. thaliana strain Columbia-0 (Col-0), which has been Sanger-sequenced and for which accurate STR genotypes are available for comparison.

For each targeted STR, we designed a MIP, which is an 80-nt oligonucleotide that contains (1) targeting arms which will uniquely hybridize to STR flanking regions; (2) a 12-nt degenerate tag to distinguish individual capture events; and (3) a common backbone for PCR and sequencing priming (Fig. 1A; Hiatt et al. 2013). In Col-0, we successfully captured all 102 STR target loci (Supplemental Fig. 3). After capture, MIPs were amplified for subsequent sequencing. Because STR amplification is prone to PCR stutter and rampant technical error, we performed optimizations, including modifying amplification conditions, specifically adjusting extension time, extension temperature, and polymerases used (see Methods).

We designed MIPs to capture $200 \mathrm{bp}$, a larger target size than is typically used (Porreca et al. 2007; Turner et al. 2009; O'Roak et al. 2012; Hiatt et al. 2013), to increase the size range of targeted STRs. This size range encompasses the majority of STRs (Gymrek et al. 2012), except those that have undergone extreme expansion as seen in some human diseases and for the intronic STR in the $A$. thaliana IIL1 gene (Gatchel and Zoghbi 2005; Sureshkumar et al. 2009). These extreme expansions are not directly accessible with MIPSTR due to our inability to capture and sequence both flanking regions. However, the systematic failure of capture and sequencing in a particular strain or individual could suggest such an expansion. Such cases can then be subjected to alternative methods such as Southern blotting or single molecule sequencing (Sureshkumar et al. 2009; Chaisson et al. 2015). Another limitation of MIP-based methods is the well-known high variability in MIP
A single-molecule Molecular Inversion Probe design

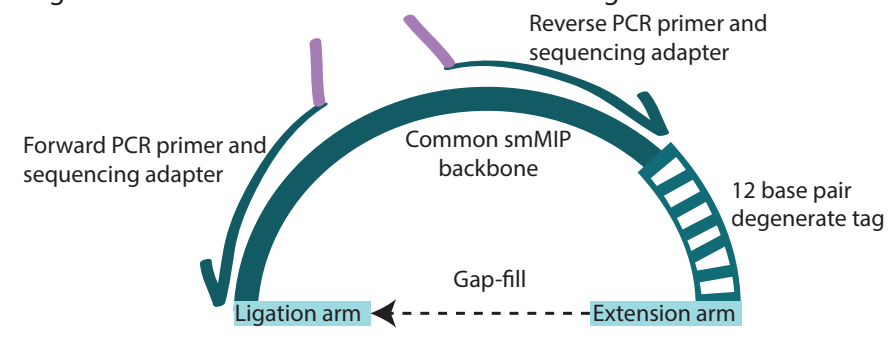

B smMIPs capture STR variation between individuals

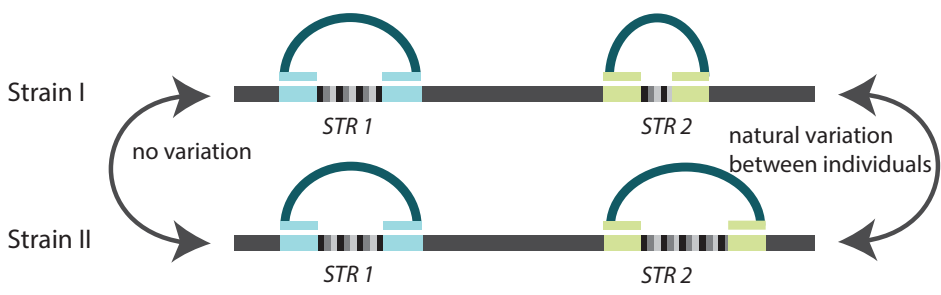

C smMIPs capture somatic STR variation within individiauls

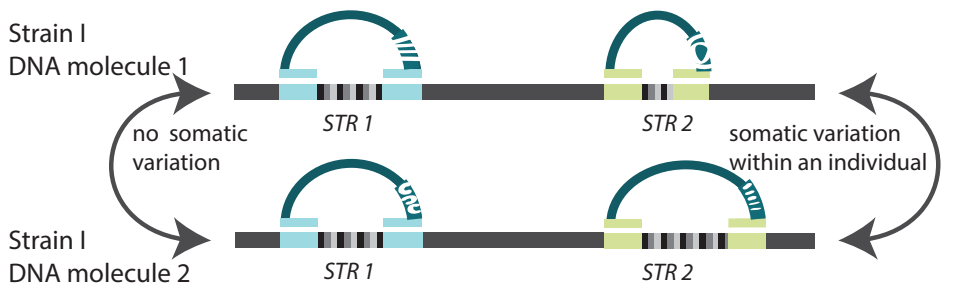

D Unique mapping strategy

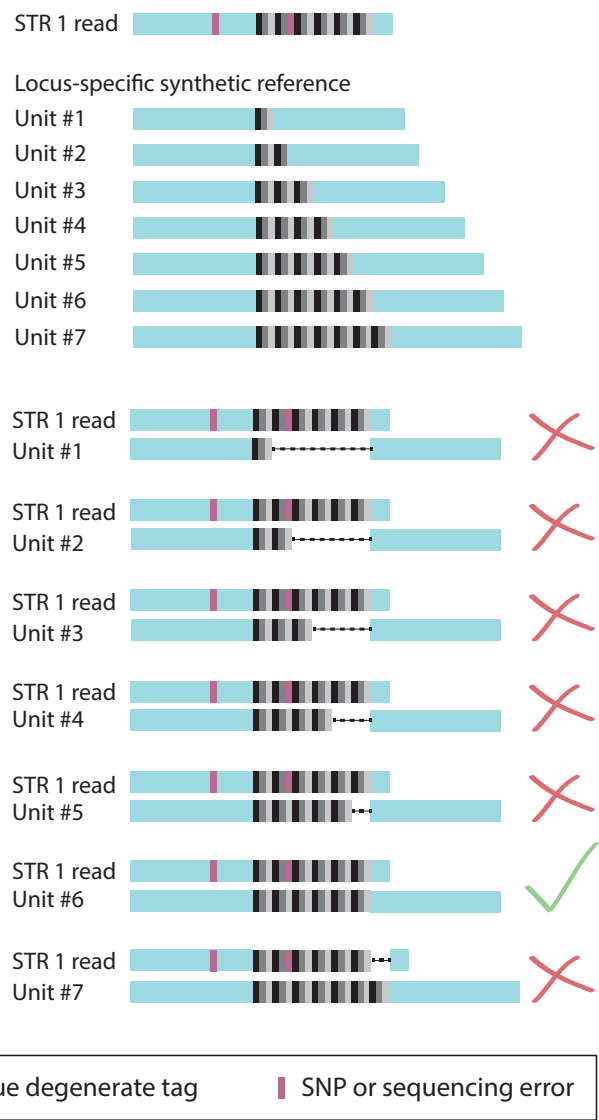

Figure 1. MIPSTR determines germline and somatic STR variation through targeted capture, sequencing, and a novel mapping strategy. ( $A$ ) Single-molecule molecular inversion probe (smMIP) with common backbone for PCR primer binding (dark-green; also shown, PCR and sequencing primers with arrows and purple sequencing adapters); 12 base pair degenerate tag (striped, green/white); and targeting arms with locus-specific, STR-flanking sequence (blue). One targeting arm is the primer for polymerase extension (extension arm). Ligation closes the circle at the other targeting arm (ligation arm). (B) Capture across genetically diverse individuals identifies germline STR variation across genetically diverse individuals. (C) MIPSTR distinguishes somatic STR variation from technical error, using many degenerate tags. STR variation within a tag-defined read group (i.e., reads with the same degenerate tag) is considered technical error. STR variation across tag-defined read groups is considered somatic variation. (D) MIPSTR maps reads from a given STR locus (based on targeting arm sequence) to its locus-specific synthetic reference with unit numbers 1-100 (1-7 shown here). The STR 1 read aligns perfectly to locus-specific synthetic reference Unit \#6 (green check mark); all other alignments show gaps (dashed line, red X). SNVs (in pink), even if occurring in the STR sequence, do not affect mapping or STR unit number genotype calls.

\section{Genome Research}

www.genome.org 
performance (Porreca et al. 2007; Turner et al. 2009; Boyle et al. 2014). MIPs with intermediate GC content in their targeting arms are most effective (O'Roak et al. 2012; Boyle et al. 2014). As in previous studies (Porreca et al. 2007; Turner et al. 2009), we observed a large dynamic range in capture efficiency among MIPS, which we attempted to correct by increasing the concentration of poorly performing MIPs (Supplemental Fig. 3). Lastly, purchasing MIPs comes at a considerable upfront cost, yet this one-time investment allows the interrogation of nearly a million genomes (see Methods). Like many genome-wide technologies, the value of MIPs and MIPSTR scales with the number of individuals assessed.

MIPSTR libraries were sequenced using 250-bp forward reads paired with 50-bp reverse reads on the Illumina MiSeq platform. The 250-bp forward reads spanned the 20 -bp ligation targeting arm followed by $200 \mathrm{bp}$ of target sequence (STR sequence and unique flanking sequence) and 20 bp extension targeting arm (large STR expansions will be missing some or all of the extension targeting arm). MIPSTR can assess STRs up to $~ 180 \mathrm{bp}$ in length, considerably longer than the STRs currently assessed from common Illumina 100-bp whole-genome-sequencing data. The 50bp reverse reads spanned the 12-bp degenerate tag, which identifies each specific MIP molecule, and the extension targeting arm (Fig. 1A). This experimental design allows MIPSTR to omit the computationally costly and error-prone step of mapping repetitive reads of low complexity to whole genomes. We sorted reads according to their MIP targeting arms, and for each MIP, used BWA (Li and Durbin 2009) to map its corresponding reads to a set of synthetic reference sequences designed specifically for each targeted STR (Fig. 1D). These synthetic references consisted of the STR sequence from the Col-0 reference genome with all possible STR unit number alleles between 1 and 100, which suffices for STR alleles within our size range. We successfully mapped $72 \%$ of all sequencing reads to the targeted loci (Table 1).

We called a genotype for each mapped read according to the quality of its alignment to an STR allele sequence (BWA alignment scores $\geq 180$ were called as genotypes). Due to our mapping strategy, variation outside of the STR or SNVs within the STR does not affect STR unit number genotype calls (Fig. 1D). For Col-0, $55 \%$ of our mappable reads yielded informative STR unit number calls. Relative to previously described methods, this result represents a dramatic improvement in the number of informative reads per unit of sequencing effort (Table 1), such that it represents a substantial improvement in the efficiency and accuracy of STR genotyping. We required at least four STR-spanning reads at each locus to call an STR genotype. Ultimately, we called unit number genotypes for 96 of the 102 examined STR target loci. For these loci, our calls were $96 \%$ concordant with the Col-0 reference allele, including the highly variable coding STR in the gene ELF3 (Fig. 2; Undurraga et al. 2012).

Most importantly, unlike any previous method that we are aware of, each STR is represented by many independent capture events of STR loci at the preamplification stage. Although amplification introduces technical error, MIPSTR distinguishes between technical error, heterozygosity, and somatic mutations by comparing reads within and between capture events (Fig. 1C). The assessment of independent capture events is enabled by the use of smMIPs with degenerate tags (Hiatt et al. 2013), i.e., the same STR locus is captured from many different cells, with each capture mediated by a MIP with a different tag. For each tag-defined read group (i.e., reads containing the same MIP tag), we assumed that the mode of called unit numbers across reads is the true allele for this capture event (Fig. 3). STR unit number variation within a tag-defined read group is considered technical error (Fig. 3; Supplemental Table 1). However, unit number variation observed among different MIP molecules, each representing independent capture events, is potentially the result of heterozygosity, somatic variation, or duplication (Figs. 1C, 3). Using the additional information of tag-defined read groups resolves the distribution of total read counts (Fig. 3, cf. A to B and C) and greatly improves confidence in STR genotype calls. Using information from tagdefined read groups also identified STR loci with consistently high technical error (Fig. 3B, middle panel), which can be excluded in subsequent analyses. Furthermore, using information from tagdefined read groups has the potential to detect multiple STR alleles within a single individual (Fig. 3B, right panel).

A. thaliana is an inbreeding plant and hence assumed to be homozygous at the vast majority of loci. Therefore, to test the potential of our method to detect multiple high-frequency alleles of the same STR, we assessed two STR loci present in two nearly identical copies on two different chromosomes in the Col-0 reference genome. For both STRs, the two genomic copies have different STR unit number genotypes in addition to SNV variation, enabling us to readily distinguish them. There was no SNV variation in the flanking regions recognized by the MIP targeting arms, allowing us to capture the duplicated loci with similar efficiency. Indeed, for both STRs, we detected both unit numbers at high levels.

Specifically, for the STR (STR ID 73a and b) with only one SNV difference between duplicate copies, we observed near equal representation of both alleles (Fig. 3B, right panel). We also observed two tag-defined read groups supporting unit number six, which may represent a somatic STR variant in this individual. Without differentiating tag-defined read groups, reads representing this STR genotype would be interpreted as technical error, like the few reads representing ELF3 STR unit number as six (Fig. 3A, cf. left panel to right panel). This example demonstrates the importance of including single-molecule information in STR genotype analysis.

Furthermore, we found evidence for the duplication of an intergenic STR that is located amid multiple transposons (Fig. 2, STR ID 89), which we confirmed using PCR. This duplication is not annotated in the Col-0 reference assembly. In theory, for duplicates (and heterozygous loci), each locus (allele) should be represented by an equal number of tag-defined read groups. In reality, this ratio deviated from the expected ratio; the more tag-defined read groups that were available for analysis, the better the results. We observed ratios as close as 49.9/50.1 (761 tag-defined read groups/764) and as far as 36/74 (40/71) for the three ascertained duplicate loci across three Col-0 siblings. These results suggest that with sufficient coverage, MIPSTR can readily identify heterozygous and somatic STR variants, which have been largely inaccessible by previous analytical or empirical methods (Gymrek et al. 2012; Guilmatre et al. 2013; Highnam et al. 2013; Duitama et al. 2014; Willems et al. 2014).

\section{MIPSTR accurately determines STR unit number genotypes across diverse $A$. thaliana strains}

We applied MIPSTR to 96 genetically diverse strains of $A$. thaliana. These strains have been assessed for over 100 quantitative phenotypes and have been previously sequenced, primarily with 36- to 64-bp reads at a coverage of $\sim 20 \times$, to detect SNVs and structural variation (Cao et al. 2011; Gan et al. 2011). STRs evolve on a different time scale than SNVs, so linkage disequilibrium between STRs and SNVs breaks down quickly (Willems et al. 2014). Therefore, we 

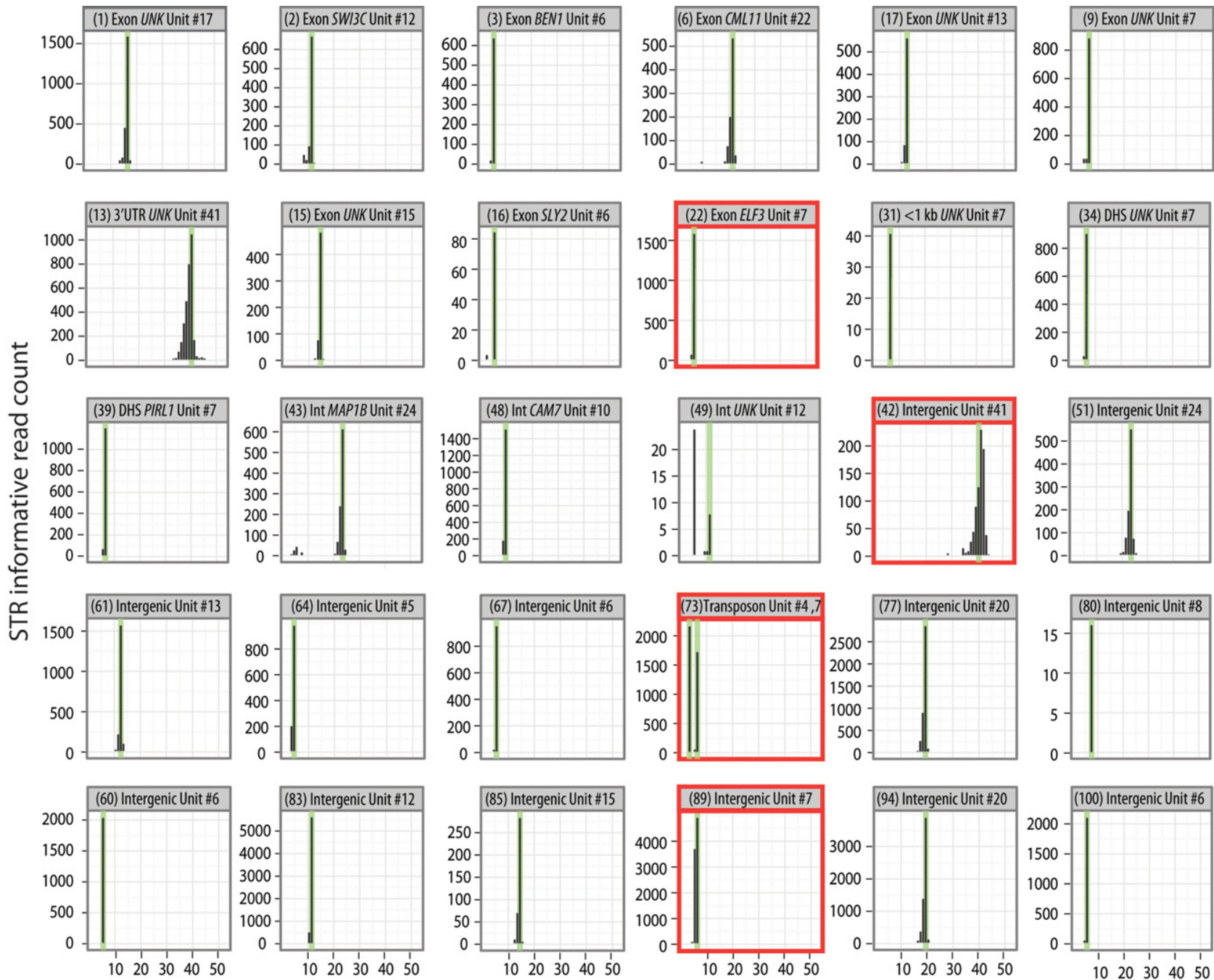

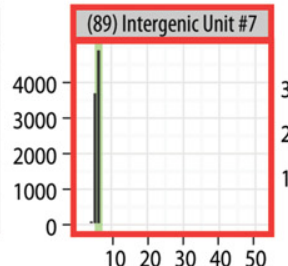

1020304050
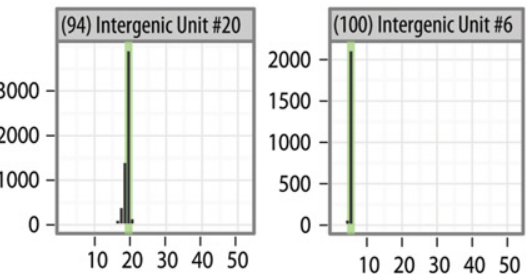

STR unit number genotype

Figure 2. MIPSTR accurately determined germline STR unit number in the reference strain Col-0. Raw read counts at 30 representative STR loci, with reference genome STR unit number indicated in green. UNK indicates gene of unknown function. Numbers shown in parentheses refer to STR IDs (see Supplemental Table 1). Two instances of genomic duplication (residing in transposons) are shown (STR ID 73 and 89); both alleles showed comparable read counts. Note that erroneous calls show low read counts or high technical error. Bold red outlines indicate examples discussed further in the text.

cannot use linked SNV data to understand the relationship between STR unit number genotype and phenotype. Given the strong potential of STR variation to cause phenotypic variation, we set out to call STR genotypes across many divergent individuals and to evaluate the contribution of STRs to the genotype-phenotype map.

We attempted to genotype 100 STRs across the 96 diverse strains of $A$. thaliana, including the reference strain Col-0, for a total of 9600 targeted STR loci in one Illumina MiSeq v2 sequencing run. MIPSTR scaled well to this task; both the number of targeted loci and the number of examined genomes can be readily increased by several orders of magnitude. STRs tend to be surrounded by repetitive sequence and AT-rich regions, but in spite of this challenge, we successfully captured STR loci genome-wide for these genetically divergent strains. Specifically, we captured at least 50 STR loci in 86 of 96 strains (90\%) (Supplemental Tables 2,3 ) and at least 75 STR loci in 59/96 strains (61\%). MIP capture efficiency across strains did not correlate with the presence of
SNPs in the targeting arms (J Fitz, S Ossowski, N Warthmann, RM Clark, K Schneeberger, and D Weigel, pers. comm.).

To apply MIPSTR to multiple strains, we pooled the 96 strainspecific capture libraries, each with a unique strain barcode on the reverse PCR primer, and sequenced as described above. For these pooled libraries, we sorted reads first by strain-specific barcode, then by targeting arm to identify the STR locus and degenerate MIP tag to identify reads originating from the same capture event (Fig. 1B,C). Similarly to our results with the reference strain Col-0, we were able to map $72 \%$ of sequence reads to their STR target loci; and of those, $64 \%$ were informative for calling STR unit number genotypes (Table 1; Supplemental Table 3). In this experiment, the Col-0 library represented $\sim 1 \%$ of the total sequence reads, which should greatly reduce the information for each STR compared to our single Col-0 library run. Despite this dramatic reduction in information content, we could accurately call germline STR unit number genotypes in Col-0 for 97\% of loci (64 of 66 loci with at least four STR-spanning reads) (Supplemental Tables 2, 3).

\section{Genome Research}

www.genome.org 
Comparing MIPSTR calls for the ELF3 STR to genotype calls from previous Sanger sequencing (Undurraga et al. 2012), MIPSTR performed with 98\% accuracy (51 of 52 strains) (Fig. 4). As previously discussed, using information from tag-defined read groups aided us in resolving STR genotypes. For example, for the strain Kin-0, total counts supported unit number 18 and 19 for the ELF3 STR (Fig. 4). Resolving read counts by tag-defined read groups enabled us to eliminate technical error and call 19 units as the correct Kin0 ELF3 STR unit number (Undurraga et al. 2012). Across all 96 strains, we called STR unit number for $60 \%$ or more of STR loci in $62 \%$ of strains, with a total of 6179 STR unit number genotypes (of 9600 targets or $\sim 64 \%$ of targets) determined with a single Illumina MiSeq v2 sequencing run. As previously shown, additional sequencing is expected to yield many more capture events and thus more complete coverage across STRs (Turner et al. 2009).

We also analyzed our data for the duplication events detected in Col-0. Of the two known duplicated STR loci, one (STR ID 64) did not show evidence for a duplication event in the $58 \mathrm{~A}$. thaliana strains with sufficient coverage (at least two tag-defined read groups) based on the presence of different STR unit numbers. However, taking SNV variation between the duplicate loci in the capture region into account (11 SNVs, present in the gap-fill region surrounding STR) (Fig. 1), we found evidence for this duplication in 38 of 58 strains. Although this duplication is presumably not recent in origin, there is no SNV variation in a given duplicate among these strains, and STR unit number variation exists only in Col-0, making it one of the least variable STRs in our data set. The second known duplication (STR ID 73) was detected in two of 14 strains with sufficient coverage. The newly identified duplication STR ID 89 was detected in 13 of 49 accessions with several STR unit number alleles across these strains. Taking SNV variation into account, we confirmed this duplication event in seven of 13 strains. Of the remaining 36 strains (i.e., strains with no difference in STR unit number but with sufficient coverage), we detected this duplication in 10 strains with SNV variation. Our results suggest that this previously unknown duplication exists in at least 23 of 49 strains.

The unit number, unit length, and purity of a given STR locus in a high-
A Total read count distributions
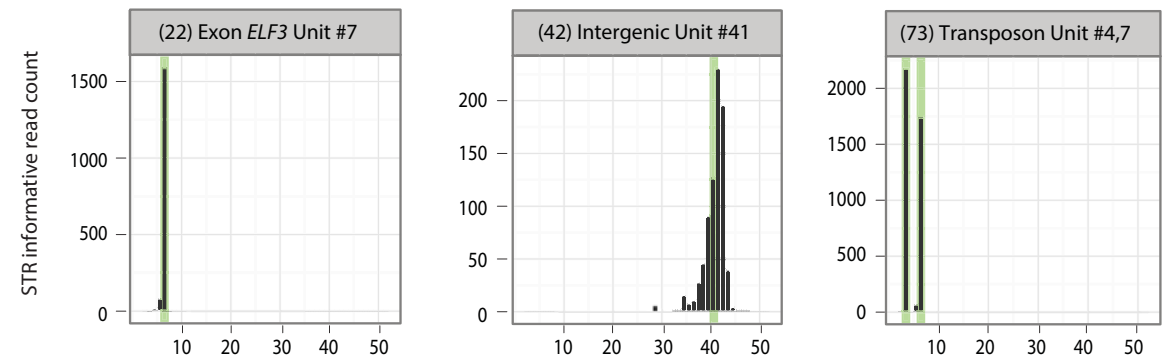

B Read counts resolved by tag-defined read groups
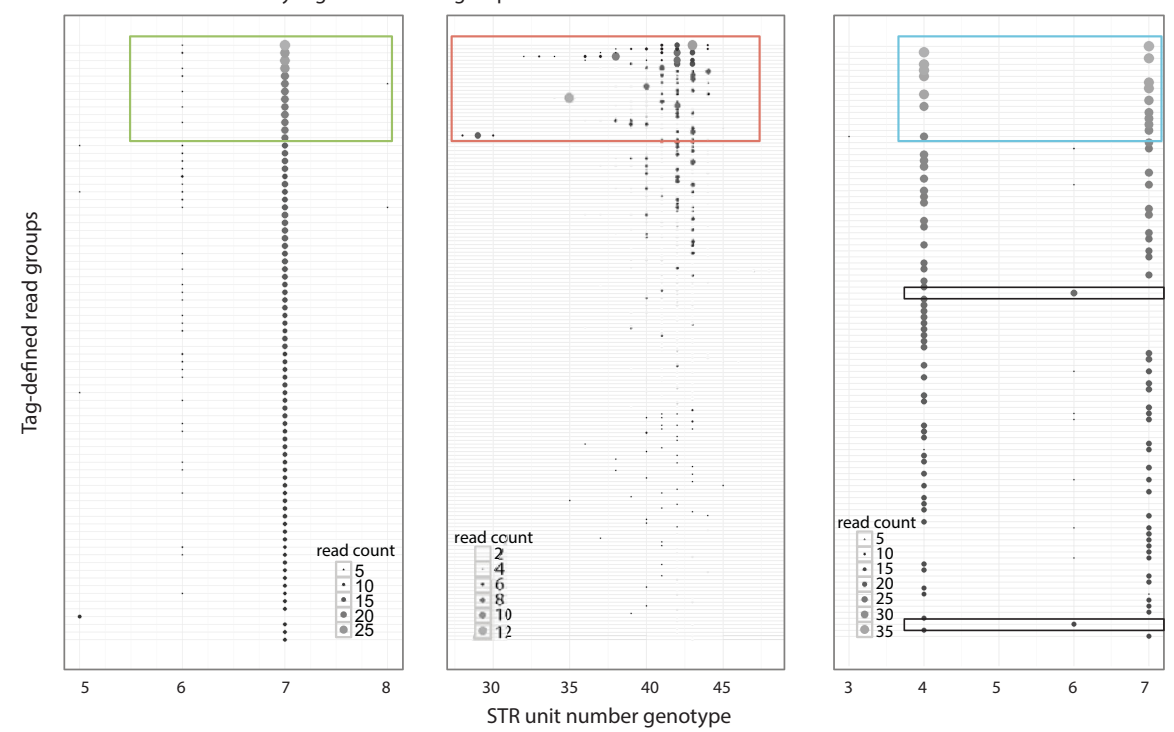

C Informative details of $B$
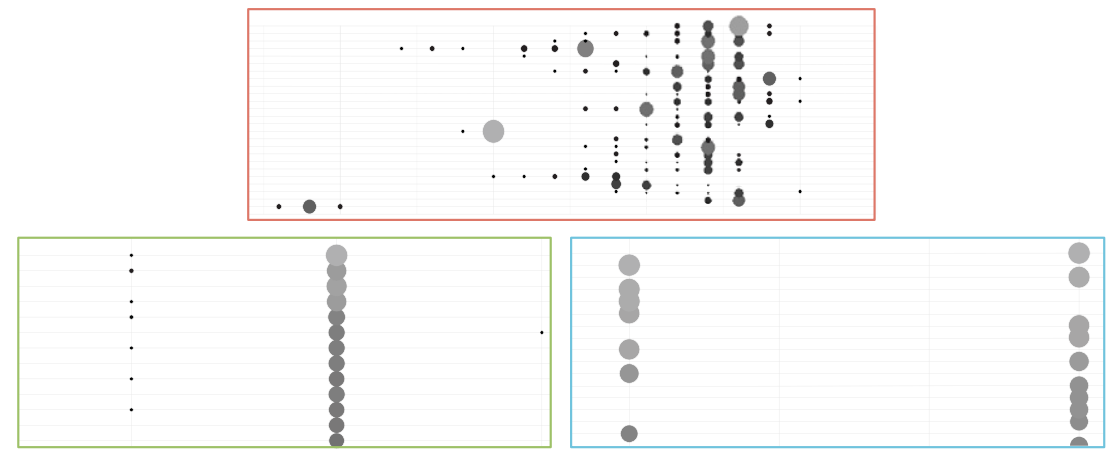

Figure 3. MIPSTR distinguished technical error from somatic variation. ( $A$ ) Three histograms from Figure 2 with total read counts. (Left) The known ELF3 STR unit number is clearly supported by the modal unit number. (Middle) This intergenic STR showed great variation in STR unit number; the mode did not support the known STR unit number. (Right) This STR resides in two copies in two different genomic locations (transposons). Both known alleles were identified, yet total read counts alone cannot distinguish genomic duplicates from technical or somatic error. $(B)$ Reads are separated into tag-defined read groups with dot sizes and shading representing read count (different scales for each locus, see inset). Colored boxes are shown in detail in C. (Left) All tag-defined read groups with one exception supported the known STR unit number seven. Most tag-defined read groups showed low levels of technical error, primarily reads with unit number six (-1), but also five and eight. (Middle) Separating reads into tag-defined read groups illustrates the extremely high technical error for this STR. The mode of a tag-defined read group was often supported by $<50 \%$ of total reads. Some tag-defined read groups contained as many as six different STR genotypes. We exclude such loci from the analysis of somatic STR variation. (Right) As expected for a duplicate STR or a heterozygote, approximately half of the tag-defined read groups support each of the known STR genotypes with very little technical error. We also observed evidence of a somatic STR allele with unit number six, which was supported by two tag-defined read groups (boxed, black outline). Note the absence of either of the known STR alleles for these tag-defined read groups. This STR genotype is also visible in the total read count histogram $(A$, right), where it would be interpreted as a technical error by other methods. (C) Detailed views of plots in $B$; outline color corresponds to respective plot. 

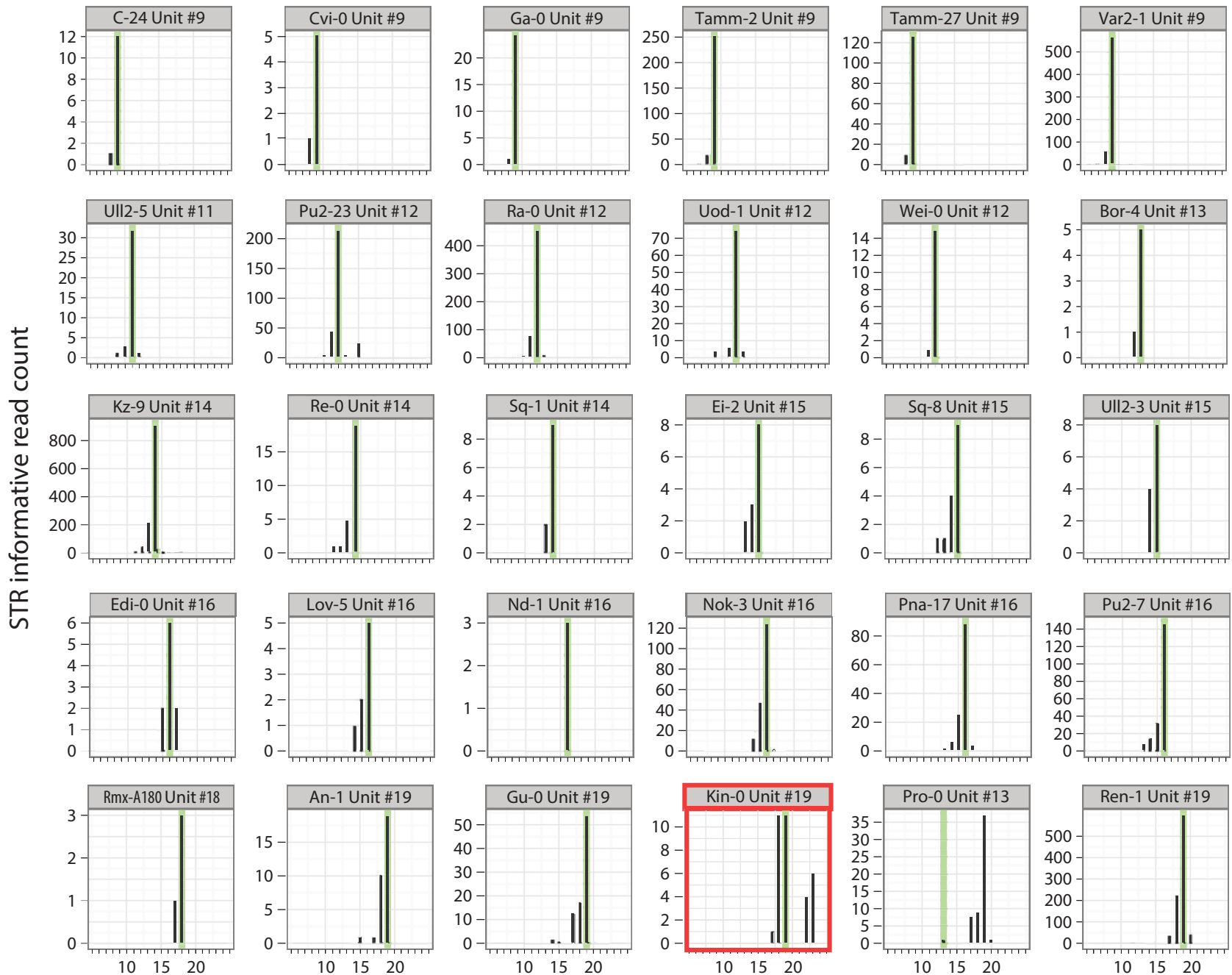

STR unit number genotype

Figure 4. MIPSTR accurately determined germline ELF3 STR unit number across genetically diverse $A$. thaliana strains. Histograms of raw read counts across 30 accessions. STR unit number as determined by Sanger sequencing is indicated in green. Using tag-defined read groups, the Kin-0 ELF3 STR genotype can be resolved to the known STR genotype even with comparatively few total reads. MIPSTR clearly calls STR unit number 19 for Pro-0. Note that different individuals of the same strain were analyzed with MIPSTR and Sanger sequencing, which may explain the discrepancy. Bold red outline indicates example discussed further in the text.

quality reference genome predict its variation across individuals (Legendre et al. 2007). STRs with high unit number, short unit length, and high purity are typically highly variable. With population-scale STR genotypes in hand, we addressed how well predicted variation of STRs (VARscore) (Supplemental Table 1; Legendre et al. 2007) correlated to observed variation across A. thaliana strains.

In general, VARscore correlated well with observed variation across STRs $(r=0.68)$ (Fig. 5), a substantially better agreement than previously observed (Duitama et al. 2014). However, this correlation was substantially weaker among coding STRs $(r=0.46)$ than among noncoding STRs $(r=0.75)$. This discrepancy suggests that sequence characteristics alone do not suffice to predict whether coding STRs vary on a population scale. Coding STRs are more likely to be functionally important, and thus are less subject to the "neutral model" of the VARscore prediction.

Deviation of predicted STR variation (i.e., VARscore) from observed variation may thus hold information about STR function and selective pressures acting upon it. Specifically, STRs that are observed to be more variable than predicted may be under diversifying selection, whereas those STRs that are observed to be less variable than predicted may be functionally constrained and under purifying selection (Press et al. 2014). For example, the STR in the gene ELF3 is highly variable across strains, ranging from seven units to as many as 29 units in a set of strains previously analyzed by Sanger sequencing (Undurraga et al. 2012). The phenotypes associated with variation in the ELF3 STR change dramatically in different genetic backgrounds, suggesting coevolution of the ELF3 STR with epistatically interacting loci (Undurraga et al. 2012). Given this STR's strong background-dependent phenotypes, it is likely under diversifying selection, and correspondingly, it is much more variable than predicted (Fig. 5).

A complementary approach for identifying STRs with important function in modulating phenotype is genome-wide association of STR genotypes with phenotypes. The standard statistical 


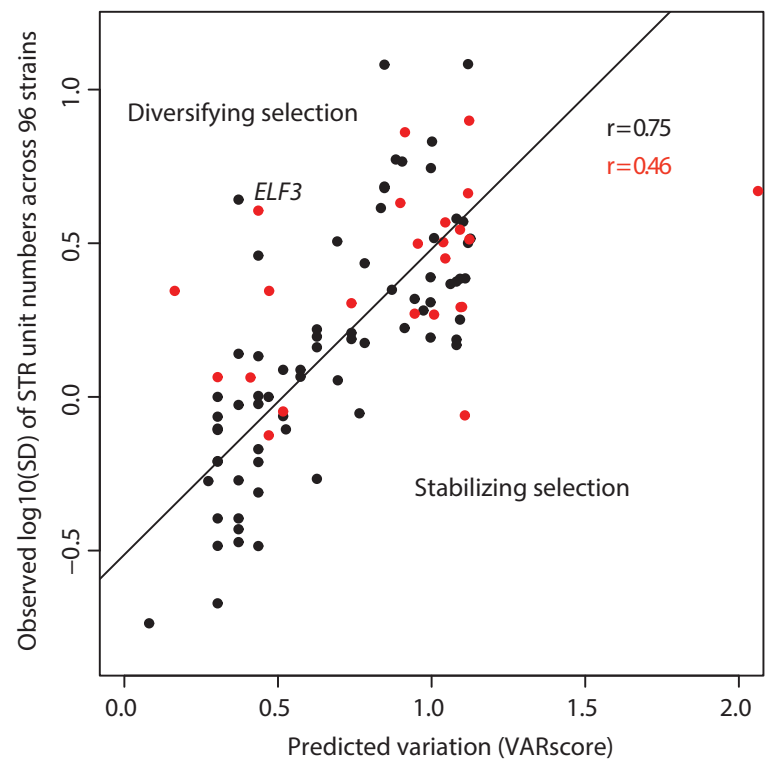

Figure 5. Observed and predicted STR variation showed greater correlation for noncoding STRs than coding STRs. The correlation between the observed $\log _{10}$ of the standard deviation of STR unit number across strains ( $y$ axis) and the VARscore ( $x$-axis), which predicts STR variation from sequence characteristics. Black points are noncoding STRs, red points are coding STRs. Outliers may indicate functional importance (ELF3 STR is indicated).

methods for associating genotype with phenotype were developed for common, biallelic SNVs (Hayes 2013). STRs are typically multiallelic and often involved in epistatic interactions, both of which make it difficult to associate STR genotype with phenotype using standard methods (Press et al. 2014). Nevertheless, we performed a naïve association analysis to determine whether STR variation across strains was associated with well-characterized phenotypes (Atwell et al. 2010). These phenotypes included morphology, developmental timing (flowering), ionomics, and gene expression, among others. We used the one-way analysis of variance (ANOVA) to detect associations between STR loci and phenotypes following previous studies (Mackay et al. 2012), modeling STR alleles as factors to avoid assumptions of linearity (Press et al. 2014). To minimize spurious associations, we excluded STRs that were typed in fewer than 10 strains from this analysis; and for each STR we excluded all strains carrying alleles present in fewer than three strains (rare alleles). We identified 124 significant associations involving 27 STRs and 41 phenotypes at a 1\% false discovery rate (Supplemental Table 4). However, an important caveat is that this analysis did not consider population structure, which is another challenge given the different evolutionary trajectories of SNVs and STRs (Willems et al. 2014). Consequently, we also performed a mixed-model analysis treating population structure (Nordborg et al. 2005) as a random effect and STR unit numbers as fixed factorial effects. Although more conservative, of the 70 associations found by this method, 56 were shared with naïve ANOVA, indicating that most variants thus identified are robust to association method (Supplemental Table 4). Furthermore, as previously observed with SNV variation, the complex trait of flowering time has many associations with variable STRs across its various potential measurements (Atwell et al. 2010).

We further investigated whether these STR-phenotype associations could be identified with common, linked SNVs (Atwell et al. 2010). For each STR-phenotype association, we identified the SNV associated with the same phenotype that is closest to the STR in question (Supplemental Table 4). No phenotype-associated STRs were linked to any SNVs associated with the same phenotype; often, associated SNVs were only found on different chromosomes. In fact, the closest $\mathrm{SNV}$ resided $>21 \mathrm{~kb}$ away from the STR associated with the same phenotype. In A. thaliana, linkage disequilibrium decays at $10 \mathrm{~kb}$ and likely decays even faster between SNVs and STRs (Nordborg et al. 2005; Kim et al. 2007; Willems et al. 2014). Thus, at least for this small set of loci, STR-phenotype associations are not captured with common SNV variation.

Our MIP-based approach can easily be scaled to thousands of targets; the human exome MIP set targets $~ 55,000$ loci (Turner et al. 2009). More than 2000 STR loci are accessible by MIPSTR in A. thaliana, and many more accessible STR loci exist in humans (Molla et al. 2009; Guilmatre et al. 2013; Duitama et al. 2014; Willems et al. 2014). Our preliminary results, considering only a fraction of the accessible $A$. thaliana STR loci, highlight the promise of STRs to contribute to the variation and heritability of quantitative traits (Press et al. 2014).

\section{MIPSTR has potential to sensitively detect heterozygous and somatic STR unit number alleles}

To determine the sensitivity with which MIPSTR detects heterozygous and somatic alleles, we mixed DNA of two divergent $A$. thaliana strains, Col-0 and Landsberg (Ler), in known ratios before MIPSTR capture and sequencing (Fig. 6). Of the 100 STR loci, 56 differed in STR unit number genotypes between Col-0 and Ler,

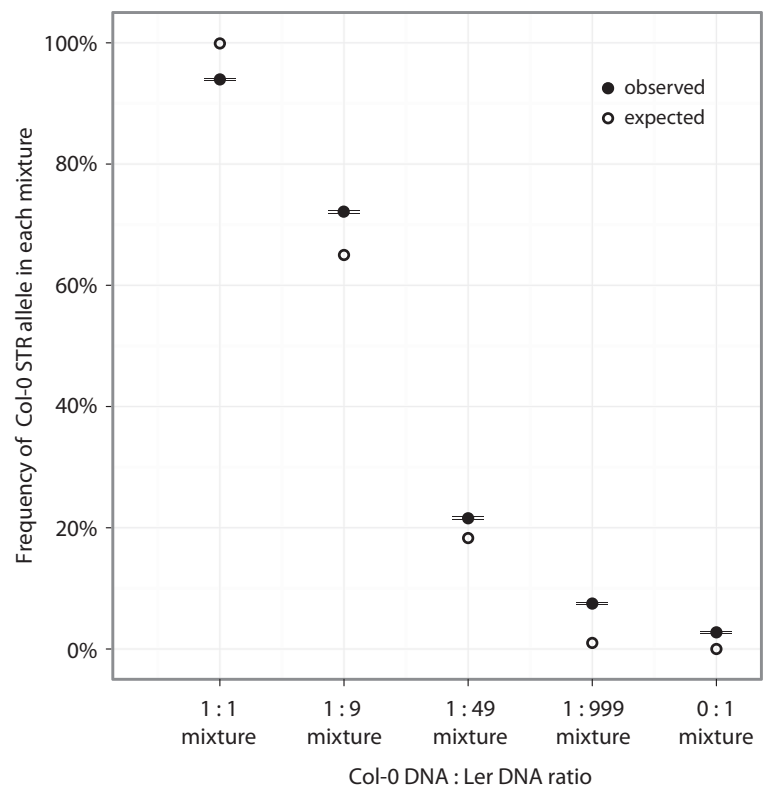

Figure 6. MIPSTR detects low frequency STR alleles. ( $x$-axis) Tested mixtures of Ler and Col-0 DNA; ( $y$-axis) probability of detecting Col-0 STR alleles; (closed circles) observed frequency of observing Col-0 STR alleles (standard error is indicated, black lines); (open circles) predicted frequency of observing Col-0 STR alleles. To calculate the observed frequency for each mixture, we resampled tag-defined read group modes supporting either the Col-0 or Ler allele at each STR locus 1000 times. The proportion of samples that carry the Col-0 allele was determined and averaged across all STR loci that differ between Ler and Col-0. To calculate the expected probability for each mixture, we assumed the known ratios of Col-0 and Ler STR alleles in each mixture and the probability of observing the Col-0 STR allele with 10 observations. 
and hence their relative presence across mixtures could be detected by MIPSTR. To assess the relative proportions of STR alleles within each mixture, we determined the number of tag-defined read groups for which the majority of reads supported either the Col0 -specific STR unit number or the Ler-specific STR unit number. This measure, however, is confounded by unequal coverage between libraries. More deeply sequenced libraries will represent a higher number of capture events per target and hence be more likely to identify rare STR alleles (i.e., somatic events). To account for variation in number of supporting tag-defined read groups per locus, we performed bootstrap resampling of the modes of the tagdefined read groups at each locus in each library 1000 times while measuring the proportion of bootstrap samples in which the Col-0 allele was detected. Applying this method to our mixing experiment, the agreement between predicted and observed frequencies of observing Col-0 STR alleles was striking. For example, when we mimicked a "heterozygous" state with a 1:1 Col-0/Ler mixture, we observed the Col-0 allele $95 \%$ of the time. This agreement of predicted and observed frequencies held across all mixtures (Fig. 6), indicating that MIPSTR sensitively detects rare alleles. Mixing one part Col-DNA into 999 parts Ler-DNA, we were able to detect the Col-alleles at half of the 56 loci.

STR instability at selected loci has been previously used as a measure of genome instability and is a hallmark of certain cancers (Boland et al. 1998; Kim et al. 2013). Our data suggest that MIPSTR has the potential to offer considerably greater resolution by assessing somatic STR variation genome-wide. To examine the potential of our method to detect decreased genome stability, we performed MIPSTR on Atmsh2 mutant plants. This mutant carries an insertion in the MSH2 gene, which is a crucial component of the DNA repair machinery. Indeed, a previous study, using a reporter system, found a $\sim 10 \%$ increase in dinucleotide STR somatic mutation events in this mutant (Golubov et al. 2010). We applied MIPSTR to three Col-0 plants and three Atmsh2 plants. After eliminating STR loci with high technical error rates (Supplemental Table 1) and loci without information for both strains, we compared the average number of STR alleles per locus with bootstrap resampling as described above. Instead of assessing two alleles, those of Col-0 and Ler as in the mixtures, we counted all alleles supported by at least one tag-defined read group in the resampling procedure. Compared to Col-0, the Atmsh 2 plants showed a $4.7 \%$ increase in average STR alleles across loci $\left(P<2.2 \times 10^{-16}\right.$, Wilcoxon test) (Supplemental Fig. 4A). Removing the two most overrepresented Col-0 and Atmsh2 libraries, (i.e., with many more tag-defined read groups represented), resulted in an even larger difference between Col-0 and Atmsh2, with a 10.6\% increase in Atmsh2 mutants' average STR alleles across all tested loci $(P<$ $2.2 \times 10^{-16}$, Wilcoxon test) (Supplemental Fig. 4B). This result is particularly remarkable considering that these loci were not optimized with respect to those most likely to exhibit somatic variation. Such optimization is readily possible with MIPSTR, for example, by applying MIPSTR to long noncoding dinucleotide STRs, which are far more prone to unit number mutation and hence somatic error. By combining such a specifically designed set of smMIPs (i.e., targets) for detecting somatic STR variation with deep sequencing, MIPSTR may be capable of identifying much more subtle increases in genome instability.

\section{Discussion}

The potential of STR variation to contribute to phenotypic variation and heritability of complex traits is increasingly recognized
(Press et al. 2014). To realize this potential, several recent efforts, relying on either analytical or experimental innovation, have made progress toward the ascertainment of accurate STR genotypes on a population scale (Gymrek et al. 2012; Guilmatre et al. 2013; Highnam et al. 2013; Cao et al. 2014; Duitama et al. 2014). However, the STR-specific challenges for accurate genotyping-mappability and high amplification stutter-were only partially addressed. Here, we resolve these challenges by capturing STRs with single-molecule Molecular Inversion Probes that allow detection of many independent capture events of the same STR across many DNA molecules (Hiatt et al. 2013). Specifically, we resolve the mappability challenge by using targeted capture and locus-specific synthetic reference sequences. We resolve the challenge of inherently high technical error in STR amplification by examining many tag-defined read groups for each STR locus. STR unit number variation within a tag-defined read group results from amplification stutter. In contrast, STR unit number variation among tag-defined read groups has the potential to detect genomic duplications, heterozygosity, and somatic variation. We show that MIPSTR is capable of distinguishing these crucial sources of STR variation within samples.

Previous studies relied on amplification of haploid or homozygous genomes to estimate technical error for STR-containing sequencing reads (Gymrek et al. 2012; Guilmatre et al. 2013; Highnam et al. 2013); this approach is confounded by somatic variation and high STR mutation rates. MIPSTR offers an experimental avenue for empirically ascertaining technical error for many types of STRs. Notably, we observed dramatic differences in technical error even among the 100 trinucleotide and hexanucleotide STRs tested here. With larger numbers and more types of STRs, one may derive more precise predictions of sequencing error based on sequence composition, length, genomic position, and other features.

However, even in this proof-of-principle study, some patterns emerged that inform our understanding of the mutability of STRs. First, as others have seen, the most common technical error we observed was the loss of one STR unit (STR variation within tag-defined read groups) (Guilmatre et al. 2013). The loss of one STR unit was also the most common somatic event (STR variation observed among tag-defined read groups). As STR variation within a tag-defined read group exclusively derives from amplification stutter, we speculate that the somatic loss of one STR unit similarly derives from amplification errors during replication rather than errors in DNA recombination or repair. Second, as anticipated by previous studies (Legendre et al. 2007), longer STRs showed both increased technical and somatic error. Third, comparing predicted (based on neutral models) to observed variation in STR unit number, we found a stronger correlation for noncoding STRs than coding STRs, consistent with greater selective pressures on the latter and suggesting that deviations from expected STR variation may hold information about an STR's functional importance.

Although the immediate application of MIPSTR is in accurately assessing germline STR variation, we also emphasize our method's potential to sensitively detect somatic STR variation. Somatic STR variation, better known as microsatellite instability (MSI), has a long history as a biomarker for certain colorectal cancers, more recently also for endometrial cancers (Boland et al. 1998; Kim et al. 2013). In fact, a recent study used exome sequencing data ( 20x coverage, 100 bp reads) (cf. Supplemental Fig. 1) to assess MSI in colorectal and endometrial tumor and matched normal samples (Kim et al. 2013). Using only STR-spanning reads, this study called an MSI event at a given STR locus by comparing STR

\section{Genome Research}

www.genome.org 
unit number distributions between tumor and matched normal samples, controlling for technical error with the STR variation observed in normal samples. As we show, comparing read distributions is vulnerable to differences in coverage and requires normalization by bootstrap resampling. MIPSTR eliminates the need to compare distributions of "normal" and "tumor" samples to correct for technical error because MIPSTR calls both germline STR genotype and somatic STR variation in a given sample.

Although the STR loci that we targeted were not optimized for somatic events, MIPSTR detected the Col-0 STR alleles even in a 1:999 mixture of Col-0 and Ler DNA. Moreover, using MIPSTR, we observed a substantial increase of somatic events in a plant mutant deficient in DNA repair. MIPSTR can readily test and identify panels of 100-500 STR loci that are particularly unstable and prone to many somatic mutation events, for example, by testing longer and less complex STRs such as di- or mononucleotides.

Beyond cancer genomics, at a population scale, somatic variation and its occurrence across tissues, developmental stages, and in response to environmental perturbations has remained largely inaccessible due to the prohibitive costs of ultra-deep and singlecell sequencing (Navin et al. 2011; Baslan et al. 2012). As STRs are highly mutable, they are arguably the best biomarkers to detect subtle perturbations of genome stability. We suggest that MIPSTR in combination with STR panels optimized for somatic variation has great promise to detect even subtle decreases in genome stability. We and others have previously proposed that subtly decreased genome stability may precede or coincide with many disease processes and may increase the penetrance of disease risk alleles (Heng 2010; Queitsch et al. 2012; Poduri et al. 2013). MIPSTR offers an approach to empirically test this hypothesis. Compared to single cell sequencing (Navin et al. 2011; Baslan et al. 2012), MIPSTR also offers a cost- and labor-efficient alternative for assessing the genetic heterogeneity of tumors, which is clinically relevant for disease treatment and prognosis (Schmitt et al. 2012; Fox et al. 2013).

Finally, we emphasize that MIPSTR is readily scalable. By simply targeting all STR loci in its size range, our method can provide genome-wide assessment of STR variation; by sequencing more deeply for an optimized panel of STR loci, our method can provide information about somatic variation. MIPSTR is applicable to any organism with a high-quality reference genome, including humans. In the future, applying MIPSTR across populations of diverse species will contribute to fulfilling the long overdue promise of STR variation for explaining trait heritability.

\section{Methods}

\section{smMIP capture reagent design}

Each smMIP is an 80-nt oligonucleotide with a 40-nt common backbone flanked by an extension arm of 16-20 nt and a ligation arm of 20-24 nt. These unique arms specifically hybridize to flanking regions of STR loci for a gap-fill of $200 \mathrm{bp}$. These targeting arms were designed based on empirical data maximizing capture efficiency of MIPs based on GC content of extension and ligation arms (O'Roak et al. 2012). We did not consider the presence of SNPs in the targeting arms in our design, but current methods include this information to minimize differences in capture efficiency across strains or between alleles (Boyle et al. 2014). Included in the $40 \mathrm{nt}$ of the common backbone are 12 random nucleotides, the degenerate tag, generating $\sim 12^{4}=1.67 \times 10^{6}$ unique sequences per MIP. The MIPs were designed for 102 STRs across the $A$. thaliana genome (Supplemental Table 1).
These MIPs were procured individually by column synthesis on a 100-nmol scale with standard desalting purification (at a cost of $\sim \$ 32$ per MIP). Once purchased, one has effectively an infinite MIP supply allowing for millions of capture reactions, justifying the considerable upfront MIP cost. Cost per MIP is significantly lower when ordering less MIP without purification (25 nmol/\$7.20 per MIP) (Hiatt et al. 2013).

MIPs were pooled at equal molarity and mixed with the target at 200-fold molar excess. The results of the first capture reaction in the Col-0 reference genome, specifically the distribution of read counts from each MIP, were used to adjust MIP concentrations. We increased the concentration of the lowest performing MIPs (28, fewest number of reads) 50 -fold; concentration of the next lowest performing group of MIPS (43) was increased 10-fold.

\section{Capture and library construction}

DNA was extracted from rosette leaves of individual 20-d-old A. thaliana plants using DNeasy Plant Maxi Kit (Qiagen). DNA was cleaned up and concentrated with Amicon Ultra Centrifugal Filter Units (Millipore).

Capture procedures were modified from previous protocols (O'Roak et al. 2012; Hiatt et al. 2013). Seven hundred fifty nanograms genomic DNA was mixed with 2 pmol smMIP mixture (starting concentration before adjustment for low performing MIPs), $1.5 \mu \mathrm{L} 10 \times$ Ampligase buffer, and molecular biology grade water to a total volume of $15 \mu \mathrm{L}$. For hybridization, these mixtures were incubated in a thermocycler with a heated lid for $10 \mathrm{~min}$ at $95^{\circ} \mathrm{C}$ followed by $48 \mathrm{~h}$ at $55^{\circ} \mathrm{C}$. After hybridization, we added 2.5 pmol dNTPs (TaKaRa), 1 unit Ex Taq polymerase (TaKaRa), $0.5 \mu \mathrm{L} 10 \times$ Ampligase buffer, 60 units Ampligase DNA ligase (Epicentre), and molecular grade water to an added volume of $5 \mu \mathrm{L}$ per mixture. The extension phase was carried out for $1 \mathrm{~h}$ at $60^{\circ} \mathrm{C}$. After gap-fill and ligation, the mixtures were cooled to $37^{\circ} \mathrm{C}$ for $2 \mathrm{~min}$. We then added 40 units of Exonuclease I (NEB) and 200 units of Exonuclease III (NEB) for a total reaction volume of $19 \mu \mathrm{L}$. To digest uncircularized and excess genomic DNA, we incubated these mixtures for $15 \mathrm{~min}$ at $37^{\circ} \mathrm{C}$, and then denatured the enzymes for $2 \mathrm{~min}$ at $92^{\circ} \mathrm{C}$.

\section{Library construction, purification, and pooling}

To create sequencing libraries, we amplified the capture reactions using a common forward primer and an indexed reverse primer. We mixed $5 \mu \mathrm{L}$ capture reaction with 12.5 pmol dNTPs (TaKaRa), $5 \mu \mathrm{L} 10 \times$ Ex Taq buffer, $25 \mu \mathrm{mol}$ forward primer, $25 \mu \mathrm{mol}$ reverse primer, 1 unit Ex Taq polymerase (TaKaRa), and molecular biology grade water to a total reaction volume of $50 \mu \mathrm{L}$. We performed an initial denaturation for $10 \mathrm{sec}$ at $98^{\circ} \mathrm{C}$, followed by 28 cycles of 10 sec at $98^{\circ} \mathrm{C}, 30 \mathrm{sec}$ at $58^{\circ} \mathrm{C}$, and $12 \mathrm{sec}$ at $72^{\circ} \mathrm{C}$. The final extension was for $3 \mathrm{~min}$ at $72^{\circ} \mathrm{C}$. PCR products were pooled as equal volumes per sample or according to gel image quantification to get approximately equal representation. We then cleaned up the pooled PCR products using AMPure XP beads (Agencourt) at $1.8 \times$ according to manufacturer's recommendations.

\section{Sequencing and primary analysis}

Samples were sequenced using the Illumina MiSeq v2 platform according to the manufacturer's instructions with custom sequencing primers (Hiatt et al. 2013). To improve cluster generation for these low complexity STR libraries, we spiked in Phi-X or whole genomic DNA libraries at 10\%-20\%. We collected one 250-bp forward read to determine sequence of the ligation arm and STR target locus, one 50-bp reverse read to determine the sequence of 
the degenerate tag and extension arm, and one 8-bp read to determine the sample index sequence. The MiSeq software sorted by index read to separate pooled libraries.

\section{Mapping and STR genotype calling}

For each target STR locus, we created a synthetic reference of 100 "chromosomes," which consisted of the Col-0 reference target sequence with 1-100 pure STR units (no SNVs). We sorted reads by the first $16 \mathrm{bp}$ of the ligation targeting arm allowing three mismatches and then used the bwasw alignment mode of the BWA aligner (Li and Durbin 2009) to map the reads to the locus-specific synthetic reference. For a given read, if the A-score of its alignment to a specific synthetic "chromosome" was $\geq 180$, we called the STR unit number of this "chromosome" for this read. Below this A-score, the read was discarded. When the sequence read ended within the STR (presumably due to a large expansion of STR units) but still mapped with an acceptable A-score, we called the genotype as $\geq$ the unit number of the "chromosome" to which the read aligned. In this way, MIPSTR can yield information about STR unit number expansions in a given individual even in the absence of STR-spanning reads. Here, these " $\geq$ " calls were not used in further analyses such as association or calculation of variation.

We then sorted the STR genotype calls by the degenerate tag on the paired reverse read from which they derived. We required an exact match of the 12-bp degenerate tag for reads to be grouped into a tag-defined read group. We then called the mode STR unit number of each tag-defined read group as the genotype of that DNA molecule. If we observed that more than one tag-defined read group supported an alternate STR allele, we considered it evidence of somatic variation.

\section{STR association with phenotypes}

We used previously published data for 107 phenotypes collected for 96 A. thaliana strains (Atwell et al. 2010). We then proceeded to detect associations between each of these phenotypes and each variable STR locus within genotyped strains. For each test, we omitted strains from the analysis that were not phenotyped for the relevant trait or genotyped at the STR in question. When considering each STR locus, we additionally omitted from analysis strains that carried STR alleles that were found in fewer than three strains total, to avoid confounding from rare alleles. We then performed one-way ANOVA to test the null hypothesis of no association between each STR and each phenotype, while treating each STR allele categorically. We chose to treat STR alleles categorically because assumptions of linearity in STR-phenotype associations are poorly founded in some cases (Undurraga et al. 2012; Press et al. 2014). Associations were accepted at a $1 \%$ false discovery rate $\left(P=1.48 \times 10^{-4}\right)$.

To take into account population structure, we used a STRUCTURE (Pritchard et al. 2000) population clustering analysis of the same 96 strains at $k=7$ from a previous study (Nordborg et al. 2005) and assigned each strain to the population group to which the largest proportion of its ancestry was attributed. We used this population assignment as a random factorial effect in a mixed-model analysis implemented in the lme4 R package (D Bates, M Machler, B Bolker, and S Walker, in prep.). To infer STRphenotype associations, we used likelihood-ratio tests to assess whether addition of a parameter representing allele identity for a specific STR improved fit relative to a model fitting only the population identity random effect. We accepted STR-phenotype associations in the mixed model at a $1 \%$ FDR.

\section{Calculating technical error rates}

To calculate the technical error rate of amplifying STR loci, we considered all tag-defined read groups for which a single STR unit number mode was supported by at least two reads. For these tagdefined read groups, we took the fraction of reads supporting unit numbers other than the mode and divided by the total number of reads. We averaged across all tag-defined read groups at a given locus for a technical error score between 0 and 1 , representing the fraction of reads at a locus known to be in error (Supplemental Table 1).

\section{Somatic allele counts}

To compare the number of somatic events occurring in different individuals, we only considered STR loci with low technical error scores (below 0.2) (Supplemental Table 1) and with information for all plants in the comparison. We used bootstrap resampling to account for sometimes vastly different read counts. For example, in the Col-0 and Ler mixing experiment, some mixture libraries had as few as 10 tag-defined read groups at a given locus. Thus, we resampled 10 modes from tag-defined read groups in these samples, counting the proportion of those samples in which the Col-0 unit number allele was present. In the Col-0 versus Atmsh2 experiment, depth of coverage was much higher, and hence we resampled 1000 modes of tag-defined read groups for each locus. For each sample, we calculated how many different STR unit number alleles were present and averaged across loci.

\section{Data access}

All raw sequencing data collected for this study have been submitted to the NCBI Sequence Read Archive (SRA; http://www.ncbi. nlm.nih.gov/sra) under accession number SRP052035.

\section{Acknowledgments}

This work was supported by grants from the National Human Genome Research Institute Interdisciplinary Training in Genomic Sciences (2T32HG35-16 to M.O.P.; T32 HG00035 to K. D.C.) and the National Institutes of Health New Innovator Award (DP2OD008371 to C.Q.). We would like to thank Matt Rich, Josh Cuperus, Matthew Snyder, Akash Kumar, Joe Hiatt, Choli Lee, Rachel Youngblood, Giang Ong, Jacob Kitzman, and members of the Queitsch laboratory for helpful discussions.

\section{References}

Atwell S, Huang YS, Vilhjálmsson BJ, Willems G, Horton M, Li Y, Meng D, Platt A, Tarone AM, Hu TT, et al. 2010. Genome-wide association study of 107 phenotypes in Arabidopsis thaliana inbred lines. Nature 465: 627-631.

Baslan T, Kendall J, Rodgers L, Cox H, Riggs M, Stepansky A, Troge J, Ravi K, Esposito D, Lakshmi B, et al. 2012. Genome-wide copy number analysis of single cells. Nat Protoc 7: 1024-1041.

Boland CR, Thibodeau SN, Hamilton SR, Sidransky D, Eshleman JR, Burt RW, Meltzer SJ, Rodriguez-Bigas MA, Fodde R, Ranzani GN, et al. 1998. A National Cancer Institute Workshop on Microsatellite Instability for cancer detection and familial predisposition: development of international criteria for the determination of microsatellite instability in colorectal cancer. Cancer Res 58: 5248-5257.

Boyle EA, O'Roak BJ, Martin BK, Kumar A, Shendure J. 2014. MIPgen: optimized modeling and design of molecular inversion probes for targeted resequencing. Bioinformatics 30: 2670-2672.

Butler AP, Trono D, Coletta LD, Beard R, Fraijo R, Kazianis S, Nairn RS. 2007. Regulation of $C D K N 2 A / B$ and Retinoblastoma genes in Xiphophorus melanoma. Comp Biochem Physiol C Toxicol Pharmacol 145: 145-155.

Cao J, Schneeberger K, Ossowski S, Günther T, Bender S, Fitz J, Koenig D, Lanz C, Stegle O, Lippert C, et al. 2011. Whole-genome sequencing of multiple Arabidopsis thaliana populations. Nat Genet 43: 956-963. 
Cao MD, Tasker E, Willadsen K, Imelfort M, Vishwanathan S, Sureshkumar S, Balasubramanian S, Bodén M. 2014. Inferring short tandem repeat variation from paired-end short reads. Nucleic Acids Res 42: e16.

Chaisson MJP, Huddleston J, Dennis MY, Sudmant PH, Malig M, Hormozdiari F, Antonacci F, Surti U, Sandstrom R, Boitano M, et al. 2015. Resolving the complexity of the human genome using singlemolecule sequencing. Nature 517: 608-611.

Chen K, Wallis JW, McLellan MD, Larson DE, Kalicki JM, Pohl CS, McGrath SD, Wendl MC, Zhang Q, Locke DP, et al. 2009. BreakDancer: an algorithm for high-resolution mapping of genomic structural variation. Nat Methods 6: 677-681.

Duitama J, Zablotskaya A, Gemayel R, Jansen A, Belet S, Vermeesch JR, Verstrepen KJ, Froyen G. 2014. Large-scale analysis of tandem repeat variability in the human genome. Nucleic Acids Res 42: 5728-5741.

Eckert KA, Hile SE. 2009. Every microsatellite is different: intrinsic DNA features dictate mutagenesis of common microsatellites present in the human genome. Mol Carcinog 48: 379-388.

Fondon JW III, Garner HR. 2004. Molecular origins of rapid and continuous morphological evolution. Proc Natl Acad Sci 101: 18058-18063.

Fox EJ, Prindle MJ, Loeb LA. 2013. Do mutator mutations fuel tumorigenesis? Cancer Metastasis Rev 32: 353-361.

Gan X, Stegle O, Behr J, Steffen JG, Drewe P, Hildebrand KL, Lyngsoe R, Schultheiss SJ, Osborne EJ, Sreedharan VT, et al. 2011. Multiple reference genomes and transcriptomes for Arabidopsis thaliana. Nature 477: 419-423.

Gatchel JR, Zoghbi HY. 2005. Diseases of unstable repeat expansion: mechanisms and common principles. Nat Rev Genet 6: 743-755.

Gemayel R, Cho J, Boeynaems S, Verstrepen KJ. 2012. Beyond junk-variable tandem repeats as facilitators of rapid evolution of regulatory and coding sequences. Genes 3: 461-480.

Golubov A, Yao Y, Maheshwari P, Bilichak A, Boyko A, Belzile F, Kovalchuk I. 2010. Microsatellite instability in Arabidopsis increases with plant development. Plant Physiol 154: 1415-1427.

Grimm D, Hagmann J, Koenig D, Weigel D, Borgwardt K. 2013. Accurate indel prediction using paired-end short reads. BMC Genomics 14: 132.

Guilmatre A, Highnam G, Borel C, Mittelman D, Sharp AJ. 2013. Rapid multiplexed genotyping of simple tandem repeats using capture and highthroughput sequencing. Hum Mutat 34: 1304-1311.

Gymrek M, Golan D, Rosset S, Erlich Y. 2012. lobSTR: a short tandem repeat profiler for personal genomes. Genome Res 22: 1154-1162.

Hajirasouliha I, Hormozdiari F, Alkan C, Kidd JM, Birol I, Eichler EE, Sahinalp SC. 2010. Detection and characterization of novel sequence insertions using paired-end next-generation sequencing. Bioinformatics 26: $1277-1283$.

Hammock EAD, Young LJ. 2005. Microsatellite instability generates diversity in brain and sociobehavioral traits. Science 308: 1630-1634.

Hayes B. 2013. Overview of statistical methods for Genome-Wide Association Studies (GWAS). Methods Mol Biol 1019: 149-169.

Heng HHQ. 2010. Missing heritability and stochastic genome alterations. Nat Rev Genet 11: 813.

Hiatt JB, Pritchard CC, Salipante SJ, O’Roak BJ, Shendure J. 2013. Single molecule molecular inversion probes for targeted, high-accuracy detection of low-frequency variation. Genome Res 23: 843-854.

Highnam G, Franck C, Martin A, Stephens C, Puthige A, Mittelman D. 2013 Accurate human microsatellite genotypes from high-throughput resequencing data using informed error profiles. Nucleic Acids Res 41: e32.

Kim S, Plagnol V, Hu TT, Toomajian C, Clark RM, Ossowski S, Ecker JR, Weigel D, Nordborg M. 2007. Recombination and linkage disequilibrium in Arabidopsis thaliana. Nat Genet 39: 1151-1155.

Kim TM, Laird PW, Park PJ. 2013. The landscape of microsatellite instability in colorectal and endometrial cancer genomes. Cell 155: 858-868.

Legendre M, Pochet N, Pak T, Verstrepen KJ. 2007. Sequence-based estimation of minisatellite and microsatellite repeat variability. Genome Res 17: 1787-1796.

Li H, Durbin R. 2009. Fast and accurate short read alignment with BurrowsWheeler transform. Bioinformatics 25: 1754-1760.

Mackay TFC, Richards S, Stone EA, Barbadilla A, Ayroles JF, Zhu D, Casillas S, Han Y, Magwire MM, Cridland JM, et al. 2012. The Drosophila melanogaster Genetic Reference Panel. Nature 482: 173-178.

Michael TP, Park S, Kim TS, Booth J, Byer A, Sun Q, Chory J, Lee K. 2007. Simple sequence repeats provide a substrate for phenotypic variation in the Neurospora crassa circadian clock. PLoS One 2: e795.

Molla M, Delcher A, Sunyaev S, Cantor C, Kasif S. 2009. Triplet repeat length bias and variation in the human transcriptome. Proc Natl Acad Sci 106: 17095-17100.
Mularoni L, Guigó R, Albà MM. 2006. Mutation patterns of amino acid tandem repeats in the human proteome. Genome Biol 7: R33.

Navin N, Kendall J, Troge J, Andrews P, Rodgers L, McIndoo J, Cook K, Stepansky A, Levy D, Esposito D, et al. 2011. Tumour evolution inferred by single-cell sequencing. Nature 472: 90-94.

Nordborg M, Hu TT, Ishino Y, Jhaveri J, Toomajian C, Zheng H, Bakker E, Calabrese P, Gladstone J, Goyal R, et al. 2005. The pattern of polymorphism in Arabidopsis thaliana. PLoS Biol 3: e196.

O'Dushlaine CT, Edwards RJ, Park SD, Shields DC. 2005. Tandem repeat copy-number variation in protein-coding regions of human genes. Genome Biol 6: R69.

O'Roak BJ, Vives L, Fu W, Egertson JD, Stanaway IB, Phelps IG, Carvill G, Kumar A, Lee C, Ankenman K, et al. 2012. Multiplex targeted sequencing identifies recurrently mutated genes in autism spectrum disorders. Science 338: 1619-1622.

Peixoto AA, Hennessy JM, Townson I, Hasan G, Rosbash M, Costa R, Kyriacou CP. 1998. Molecular coevolution within a Drosophila clock gene. Proc Natl Acad Sci 95: 4475-4480.

Poduri A, Evrony GD, Cai X, Walsh CA. 2013. Somatic mutation, genomic variation, and neurological disease. Science 341: 1237758.

Porreca GJ, Zhang K, Li JB, Xie B, Austin D, Vassallo SL, LeProust EM, Peck BJ, Emig CJ, Dahl F, et al. 2007. Multiplex amplification of large sets of human exons. Nat Methods 4: 931-936.

Press M, Carlson KD, Queitsch C. 2014. The overdue promise of short tandem repeat variation for heritability. bioRxiv doi: 10.1101/006387.

Pritchard JK, Stephens M, Donnelly P. 2000. Inference of population structure using multilocus genotype data. Genetics 155: 945-959.

Qi J, Zhao F. 2011. in GAP-sv: a novel scheme to identify and visualize structural variation from paired end mapping data. Nucleic Acids Res 39: W567-W575.

Queitsch C, Carlson KD, Girirajan S. 2012. Lessons from model organisms: phenotypic robustness and missing heritability in complex disease. PLoS Genet 8: e1003041.

Rosas U, Mei Y, Xie Q, Banta JA, Zhou RW, Seufferheld G, Gerard S, Chou L, Bhambhra N, Parks JD, et al. 2014. Variation in Arabidopsis flowering time associated with cis-regulatory variation in CONSTANS. Nat Commun 5: 3651.

Sawyer LA, Hennessy JM, Peixoto AA, Rosato E, Parkinson H, Costa R, Kyriacou CP. 1997. Natural variation in a Drosophila clock gene and temperature compensation. Science 278: 2117-2120.

Scarpino SV, Hunt PJ, Garcia-De-Leon FJ, Juenger TE, Schartl M, Kirkpatrick M. 2013. Evolution of a genetic incompatibility in the genus Xiphophorus. Mol Biol Evol 30: 2302-2310.

Schmitt MW, Prindle MJ, Loeb LA. 2012. Implications of genetic heterogeneity in cancer. Ann NY Acad Sci 1267: 110-116.

Sullivan AM, Arsovski AA, Lempe J, Bubb KL, Weirauch MT, Sabo PJ, Sandstrom R, Thurman RE, Neph S, Reynolds AP, et al. 2014. Mapping and dynamics of regulatory DNA and transcription factor networks in A. thaliana. Cell Rep 8: 2015-2030.

Sureshkumar S, Todesco M, Schneeberger K, Harilal R, Balasubramanian S, Weigel D. 2009. A genetic defect caused by a triplet repeat expansion in Arabidopsis thaliana. Science 323: 1060-1063.

Tae H, McMahon KW, Settlage RE, Bavarva JH, Garner HR. 2013. ReviSTER: an automated pipeline to revise misaligned reads to simple tandem repeats. Bioinformatics 29: 1734-1741.

Turner EH, Lee C, Ng SB, Nickerson DA, Shendure J. 2009. Massively parallel exon capture and library-free resequencing across 16 genomes. Nat Methods 6: 315-316.

Undurraga SF, Press MO, Legendre M, Bujdoso N, Bale J, Wang H, Davis SJ, Verstrepen KJ, Queitsch C. 2012. Background-dependent effects of polyglutamine variation in the Arabidopsis thaliana gene ELF3. Proc Nat Acad Sci 109: 19363-19367.

Verstrepen KJ, Jansen A, Lewitter F, Fink GR. 2005. Intragenic tandem repeats generate functional variability. Nat Genet 37: 986-990.

Willems TF, Gymrek M, Highnam G, The 1000 Genomes Project Consortium, Mittelman D, Erlich Y. 2014. The landscape of human STR variation. bioRxiv doi: 10.1101/004671.

Yu F, Sabeti PC, Hardenbol P, Fu Q, Fry B, Lu X, Ghose S, Vega R, Perez A, Pasternak S, et al. 2005. Positive selection of a pre-expansion CAG repeat of the human SCA2 gene. PLoS Genet 1: e41.

Received July 25, 2014; accepted in revised form February 5, 2015. 


\section{Corrigendum}

Genome Research 25: 750-761 (2015)

\section{Corrigendum: MIPSTR: a method for multiplex genotyping of germline and somatic STR variation across many individuals}

Keisha D. Carlson, Peter H. Sudmant, Maximilian O. Press, Evan E. Eichler, Jay Shendure, and Christine Queitsch

Five measures of reagents reported in the Methods section of the paper are incorrect. When mixing 750 ng genomic DNA with the MIPs mixture, we added $2 \mathrm{fmol}$ of MIPs mixture ( $1 \mu \mathrm{L}$ of $2 \mathrm{nM}$ MIPs mixture), not 2 pmol as stated in the text. In the extension and ligation reaction, we added $2.5 \mathrm{nmol} \mathrm{dNTPs}(1 \mu \mathrm{L}$ of $2.5 \mathrm{mM} \mathrm{dNTPs})$, not $2.5 \mathrm{pmol}$. After the exonuclease was added, the final reaction volume was $24 \mu \mathrm{L}$, not $19 \mu \mathrm{L}$. Finally, during the PCR reaction of library construction, we added $12.5 \mathrm{nmol}$ of dNTPs ( $5 \mu \mathrm{L}$ of $2.5 \mathrm{mM} \mathrm{dNTP})$ and $25 \mathrm{pmol}$ of the forward and reverse primers ( $5 \mu \mathrm{L}$ of $5 \mu \mathrm{M}$ stock solution of each forward and reverse primer), not $12.5 \mathrm{pmol}$ of dNTPs and $25 \mu \mathrm{mol}$ primers as reported.

The authors thank Joseph Christopher and Doug Winton for using the method and pointing out these errors. These errors do not affect any of the conclusions of the paper, but could prevent others from successfully using the method. The authors sincerely apologize for any inconvenience these errors may have caused.

doi: 10.1101/gr.195115.115 


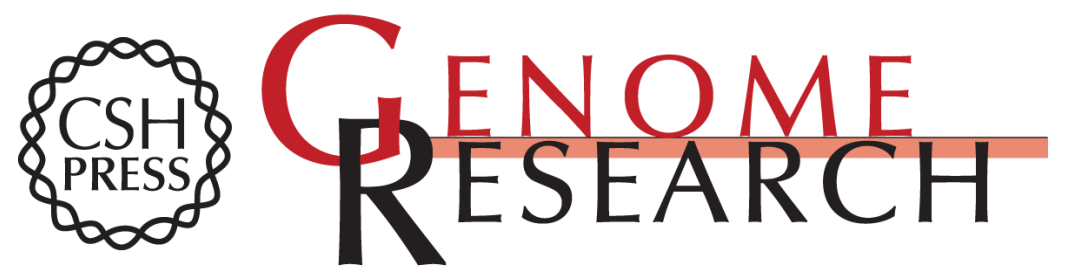

\section{MIPSTR: a method for multiplex genotyping of germline and somatic STR variation across many individuals}

Keisha D. Carlson, Peter H. Sudmant, Maximilian O. Press, et al.

Genome Res. 2015 25: 750-761 originally published online February 6, 2015

Access the most recent version at doi:10.1101/gr.182212.114

\section{Supplemental http://genome.cshlp.org/content/suppl/2015/02/24/gr.182212.114.DC1 \\ Material}

Related Content Corrigendum: MIPSTR: a method for multiplex genotyping of germline and somatic STR variation across many individuals

Keisha D. Carlson, Peter H. Sudmant, Maximilian O. Press, et al.

Genome Res. August, 2015 25: 1244

References This article cites 57 articles, 17 of which can be accessed free at:

http://genome.cshlp.org/content/25/5/750.full.html\#ref-list-1

Articles cited in:

http://genome.cshlp.org/content/25/5/750.full.html\#related-urls

Creative This article is distributed exclusively by Cold Spring Harbor Laboratory Press for the Commons first six months after the full-issue publication date (see

License

http://genome.cshlp.org/site/misc/terms.xhtml). After six months, it is available under a Creative Commons License (Attribution-NonCommercial 4.0 International), as described at http://creativecommons.org/licenses/by-nc/4.0/.

Email Alerting Receive free email alerts when new articles cite this article - sign up in the box at the Service top right corner of the article or click here.

\section{Affordable, Accurate Sequencing.}

To subscribe to Genome Research go to:

https://genome.cshlp.org/subscriptions 